\title{
Prevalência e suscetibilidades bacterianas das infecções comunitárias do trato urinário, em Hospital Universitário de Brasília, no período de 2001 a 2005
}

\author{
Prevalence and bacterial susceptibility of community acquired urinary \\ tract infection in University Hospital of Brasília, 2001 to 2005
}

\author{
Marcelle Cristina da Silva Pires ${ }^{1}$, Karla de Sousa Frota ${ }^{1}$, Paulo de Oliveira Martins Junior ${ }^{2}$, \\ Amabel Fernandes Correia ${ }^{2}$, Juan José Cortez-Escalante ${ }^{3}$ e Celeste Aída Silveira ${ }^{4}$
}

\begin{abstract}
RESUMO
A infecção do trato urinário é uma das afecções mais comuns da clínica médica, sendo mandatório o conbecimento epidemiológico da mesma e do perfil de sensibilidade dos agentes etiológicos. O estudo teve como objetivo identificar os agentes etiológicos mais freqüentes e o perfil de sensibilidade aos antimicrobianos das bactérias isoladas de uroculturas de pacientes ambulatoriais atendidos no Hospital Universitário de Brasília no período de 2001 a 2005. Foram analisadas 2.433 uroculturas positivas realizadas no laboratório de microbiologia do Hospital Universitário de Brasília. A Escherichia coli foi a bactéria mais isolada (62,4\%), seguida de Klebsiella pneumoniae (6,8\%) e Proteus mirabillis (4,7\%). A Escherichia coli apresentou maior sensibilidade à amicacina (98,6\%), gentamicina (96,2\%), nitrofurantoína (96,3\%), e às quinolonas ciprofloxacina (90,9\%) e norfloxacina (89,8\%), com baixa sensibilidade ao sulfametoxazol-trimetoprima (50,6\%). As outras bactérias apresentaram similar padrão de sensibilidade. Em conclusão, a Escherichia coli foi a bactéria mais isolada, sendo altamente sensível aos amiglicosídeos, nitrofurantoína e quinolonas.
\end{abstract}

Palavras-chaves: Infecção do trato urinário. Urocultura. Antimicrobianos. Sensibilidade.

\section{ABSTRACT}

Urinary tract infection is among the most common infectious diseases in clinical medicine, and knowledge of its epidemiology and the sensitivity profile of the etiological agents is mandatory. The aim of this study was to identify the most frequent etiological agents and the profile of sensitivity to antimicrobial agents of the bacteria isolated from urine cultures from outpatients at the University Hospital of Brasilia between 2001 and 2005. From analyses at the hospital's microbiology laboratory, there were 2,433 positive urine cultures. Escherichia coli was the most commonly isolated bacteria (62.4\%), followed by Klebsiella pneumoniae (6.8\%) and Proteus mirabilis (4.7\%). Escherichia coli showed the highest sensitivity to amikacin (98.6\%), gentamicin (96.2\%), nitrofurantoin (96.3\%) and the quinolones ciprofloxacin (90.9\%) and norfloxacin (89.8\%), with low sensitivity to sulfamethoxazole-trimethoprim (50.6\%). The others bacteria presented similar sensitivity profiles. In conclusion, Escherichia coli was the most commonly isolated bacteria, and it was highly sensitive to aminoglycosides, nitrofurantoin and quinolones.

Key-words: Urinary tract infection. Urine culture. Antimicrobials. Sensitivity.

A infecção do trato urinário (ITU) é uma das afecções mais comuns da clínica médica ${ }^{9}$, figurando como a segunda infecção mais comum no ser humano ${ }^{21}$. Embora predomine entre as mulheres ${ }^{6}$, é também freqüente em homens nas faixas etárias extremas da vida.

A infecção do trato urinário é definida como a invasão microbiana de qualquer órgão do trato urinário, desde a uretra até 0 rins ${ }^{10}$, podendo atingir inclusive estruturas adjacentes. Pode cursar com ou sem sintomas, nesse caso sendo conhecida como bacteriúria assintomática 5 .

0 trato urinário pode ser invadido por uma grande diversidade de microrganismos, como bactérias, vírus e fungos ${ }^{2}$. A etiologia da grande maioria dos casos corresponde à infecção por bactérias Gram-negativas, sendo a Escherichia coli o microrganismo invasor mais comum ${ }^{19}$.

\footnotetext{
1. Curso de Medicina, Universidade de Brasília, Brasília, DF. 2. Laboratório de Microbiologia, Hospital Universitário de Brasília, Brasília, DF. 3. Núcleo de Medicina Tropical, Universidade de Brasília, Brasília, DF. 4. Disciplina de Doenças Infecciosas e Parasitárias, Universidade de Brasília, Brasília, DF.

Endereço para correspondência: Dr ${ }^{a}$ Marcelle Cristina da Silva Pires. SQN 407, Bloco P, Apt $^{0}$ 307, 70855-160 Brasília, DF.

Tel: $55619631-6855$

e-mail: m0338460@aluno.unb.br

Recebido para publicação em: 21/06/2007

Aceito em: 11/10/2007
} 
Quando sintomática, o quadro clínico de ITU pode ser bastante sugestivo para o diagnóstico, incluindo disúria, polaciúria, dor lombar e/ou suprapúbica, febre e calafrios (na pielonefrite), urgência miccional, nictúria, urina turva (pela presença de piúria) e/ou avermelhada (pela hematúria). Contudo, o diagnóstico só é confirmado pela urocultura, considerada o padrão-ouro no diagnóstico de ITU ${ }^{18}$. A urocultura quantitativa não só indica a ocorrência de multiplicação bacteriana no trato urinário, como também permite o isolamento do agente etiológico e o estudo de sua sensibilidade aos antimicrobianos através do antibiograma ${ }^{16}$. A infecção urinária é caracterizada pelo crescimento de, no mínimo, 100 mil unidades formadoras de colônia (UFC) por mililitro de urina colhida em jato médio e de maneira asséptica. Apesar de bastante preciso, esse exame apresenta certas desvantagens, como a demora na obtenção do resultado e o alto custo ${ }^{3}$.

0 conhecimento epidemiológico das ITU e do padrão de sensibilidade/resistência dos agentes causais cresce em importância diante da falha no tratamento, que na maioria das vezes é empírico, sendo que o teste de sensibilidade a antimicrobianos orienta a nova conduta terapêutica.

A prevalência de resistência bacteriana aos antibióticos nas infecções comunitárias vem crescendo, mas, ao contrário das infecções nosocomiais, há poucos trabalhos publicados que levantam essa questão ${ }^{19}$. Esse crescente aumento de bactérias resistentes a múltiplos antimicrobianos representa um desafio no tratamento das infecções, necessitando, portanto, de revisões e análises periódicas.

Por essas considerações, o presente trabalho tem por objetivo identificar os agentes etiológicos mais frequientes e o perfil de sensibilidade aos antimicrobianos das bactérias isoladas de uroculturas de pacientes ambulatoriais atendidos no Hospital Universitário de Brasília (HUB) no período de 2001 a 2005, com o intuito de conhecer melhor nossa casuística e tentar otimizar o manejo clínico de nossos pacientes com ITU.

\section{MATERIAL E MÉTODOS}

Foi feito um levantamento de todas as uroculturas positivas (a partir de $100 \mathrm{mil} \mathrm{UFC/ml)} \mathrm{de} \mathrm{pacientes} \mathrm{ambulatoriais,} \mathrm{realizadas}$ no laboratório de microbiologia do HUB no período de 2001 a 2005.

A urina dos pacientes foi coletada pela técnica do jato médio, em frascos assépticos, pelo menos 2 horas após a última micção. As uroculturas foram realizadas com semeadura em alça calibrada (1:1.000) em agar cled e agar azida, após homogeneização da urina. 0 material foi incubado em estufa bacteriológica $\left(35-1^{\circ} \mathrm{C}\right)$ por 18 a 24 horas, e posteriormente analisado, tendo os resultados transcritos nos livros de registro do laboratório ${ }^{1}$. Após identificação do microrganismo, realizavam-se os antibiogramas através do método de difusão de disco em ágar Müeller-Hinton ${ }^{4}$. Os discos com antibióticos eram distribuídos nas placas, as quais eram incubadas por 24 horas. A determinação do grau de sensibilidade bacteriana levou em consideração o diâmetro do halo seguindo orientações do National Committee for Clinical Laboratory Standards ${ }^{11}$.
Os dados foram coletados dos livros de registro do laboratório de microbiologia, descartando-se as duplicatas, isto é, exames de um mesmo paciente, com o mesmo agente etiológico e o mesmo perfil de sensibilidade dentro de um período de quatro meses. A análise dos dados foi feita com o programa Statistical Package for the Social Sciences (SPSS), versão $13^{12}$. A variação da sensibilidade da Escherichia coli aos antimicrobianos, ao longo dos anos, foi analisada pelo teste do chi-quadradro $\left(\mathrm{X}^{2}\right)$, sendo considerado estatisticamente significativo $\mathrm{p}<0,05$ (95\%). Todas as análises foram realizadas com grau de liberdade igual a quatro.

\section{RESULTADOS}

Os dados foram coletados do livro de registro do Laboratório de Microbiologia do Hospital Universitário de Brasília; no entanto, em muitos registros faltavam informações acerca do sexo, idade e clínica do paciente. Devido a essas limitações, os dados serão analisados sem especificações de gênero, idade ou clínica.

No período de 2001 a 2005 , foram registradas 2.433 uroculturas positivas. A bactéria Escherichia coli foi o agente mais freqüente, representando 62,4\% do total (1.517 ocorrências nos últimos cinco anos), seguida pela Klebsiella pneumoniae (6,8\%), Proteus mirabillis (4,7\%), Pseudomonas aeruginosa $(2,9 \%)$, Enterobacter aerogenes (2,5\%), Proteus vulgaris (2,2\%) e Staphylococcus saprophyticus (2\%). Outros agentes constituíram 16,6\% da amostra, sendo eles: Staphylococcus aureus, Staphylococcus epidermidis, Enterobacter cloacae, Enterobacter faecalis, Citrobacter freundi, Klebsiella oxitoca, Enterobacter gergoviae, Streptococcus agalactiae, Staphylococcus aureus meticilina-resistente (MRSA), Citrobacter diversus, Pantoea agglomerans, Morganella morganii, Proteus penneri, Acinetobacter baumanii, Klebsiella ozaenae, Enterobacter aglomerans, Serratia marcescens, Burkholderia cepacia, Edwardsiella tarda, Providencia rettgeri, Enterococcus sp, Serratia liquefaciens, Pseudomonasputida, Pseudomonas fluorescens, Acinetobacter calcoaceticus, Streptococcus viridans, Enterobacter sakazaki, Stenotrophomonas maltophilia, Pseudomonas stutzeri, Chromobacterium violaceum, Yersinis enterocolitica $e$ Shigella sp (Figura 1).

No período estudado, a bactéria Escherichia coli apresentou ótima sensibilidade à amicacina (98,6\%), gentamicina (96,2\%) e nitrofurantoína (96,3\%). Obteve menor sensibilidade, mas ainda próxima a 90\%, às quinolonas ciprofloxacina $(90,9 \%)$ e norfloxacina (89,8\%). A associação sulfametoxazol-trimetoprima (STX-TMP), um dos antimicrobianos mais usados na prática clínica do nosso serviço, apresentou sensibilidade muito baixa (50,6\%) (Figura 2).

Os aminoglicosídeos mostraram-se muitos ativos frente à Escherichia coli; a amicacina em nenhum ano apresentou sensibilidade menor que 98\%, e a gentamicina apresentou a menor (93,2\%) sensibilidade no ano 2003. A nitrofurantoína também mostrou boa atividade contra a Escherichia coli durante 


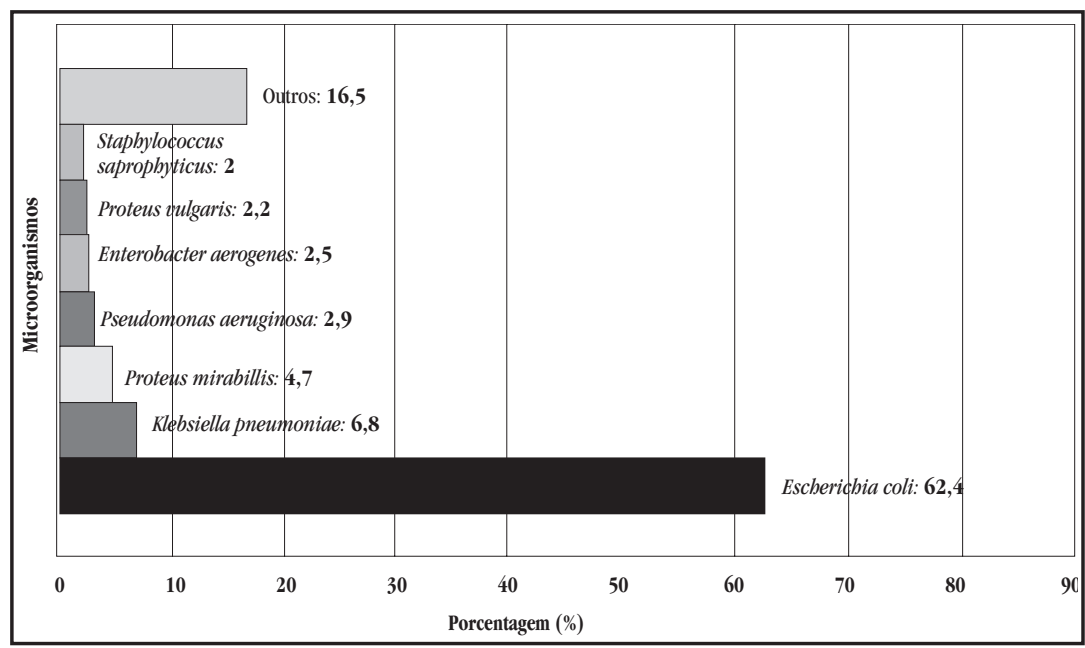

Figura 1 - Agentes patogênicos mais prevalentes nas infecções do trato urinário de pacientes ambulatoriais do Hospital Universitário de Brasília de 2001 a 2005.

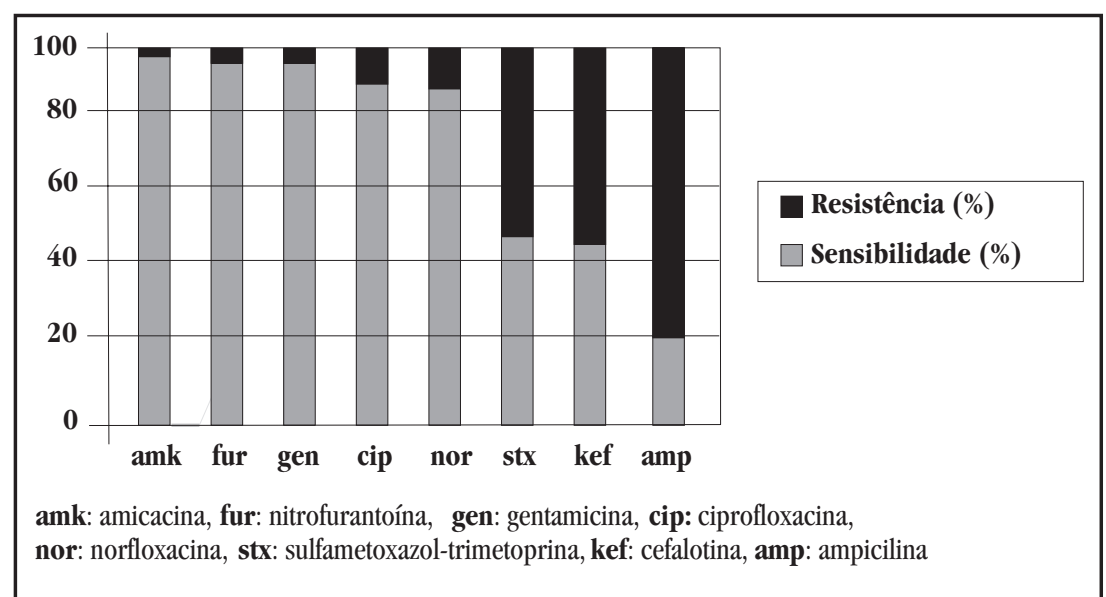

Figura 2 - Perfil de sensibilidade da Escherichia coli aos antimicrobianos usados nas infecções do trato urinário de pacientes ambulatoriais atendidos no Hospital Universitário de Brasília de 2001 a 2005.

todo o período estudado, com a menor $(95,2 \%)$ sensibilidade em 2001. Desde o ano 2001, as quinolonas mostram uma leve queda na atividade contra a Escherichia coli; as sensibilidades do microrganismo à ciprofloxacina e norfloxacina foram 95 e 95,5\%, respectivamente; não obstante, para o ano 2005, diminuíram a 88,4 e 85,5\%, respectivamente. Apesar disso, não houve diferença significativa frente à ciprofloxacina ao longo dos 5 anos. Na avaliação da cefalotina, a Escherichia coli mostrou sensibilidade intermediária $(75,7 \%)$ no ano 2005 , sendo a melhor porcentagem desde 2001. A grande diferença observada na sensibilidade frente à cefalotina e à ampicilina nos anos 2001 e 2002 deveu-se ao fato de poucos antibiogramas terem testado esses antibióticos quando comparados às amostras dos demais anos. A sulfametoxazol-trimetoprima apresentou baixa atividade frente à Escherichia coli, 62\% de sensibilidade no ano $2002 \mathrm{e}$ $50 \%$ nos últimos dois anos da avaliação. Frente à ampicilina, esse microrganismo mostrou as piores sensibilidades, sendo 38\% no ano 2005 (Tabela 1).

Ao longo dos cinco anos de estudo, houve variação estatisticamente significativa $(\mathrm{p}<0,05)$ da sensibilidade aos antimicrobianos gentamicina, norfloxacina, STX-TMP e ampicilina. Contudo, a sensibilidade da gentamicina e norfloxacina se manteve sempre acima de $85 \%$, enquanto a sensibilidade frente à ampicilina e STX-TMP sempre se mostrou insatisfatória (máximo de 62\% para STX-TMP). Os demais antimicrobianos não mostraram variação significativa ao longo dos anos. A análise estatística da cefalotina e da ampicilina foi feita considerando-se apenas os três últimos anos, pelo motivo já exposto.

As bactérias Klebsiella pneumoniae, Proteus mirabillis, Pseudomonas aeruginosa e Enterobacter aerogenes mostraram sensibilidade variável aos antimicrobianos avaliados. Semelhantemente à Escherichia coli, a Klebsiella pneumoniae foi altamente sensível aos aminoglicosídeos e quinolonas durante todo o período avaliado, assim como Proteus mirabillis e Enterobacter aerogenes. No entanto, a Pseudomonas aeruginosa mostrou sensibilidade intermediária frente a esses antimicrobianos; para o ano 2005, a amicacina, gentamicina, ciprofloxacina e norfloxacina apresentaram 75\%; 58,3\%; 58,3\% e $61,5 \%$ de atividade (Tabela 2 ). 
Tabela 1 - Evolução do perfil de sensibilidade da Escherichia coli aos antimicrobianos usados nas infecções do trato urinário de pacientes ambulatoriais atendidos no Hospital Universitário de Brasília de 2001 a 2005.

\begin{tabular}{|c|c|c|c|c|c|c|c|c|c|c|c|c|c|}
\hline \multirow[t]{2}{*}{ Antimicrobianos } & \multicolumn{2}{|c|}{ Total } & \multicolumn{2}{|c|}{2001} & \multicolumn{2}{|c|}{2002} & \multicolumn{2}{|c|}{2003} & \multicolumn{2}{|c|}{2004} & \multicolumn{2}{|c|}{2005} & \multirow[b]{2}{*}{ Valor de $p$} \\
\hline & $\mathrm{n}^{0}$ & $\%$ & $\mathrm{n}^{0}$ & $\%$ & $\mathrm{n}^{0}$ & $\%$ & $\mathrm{n}^{0}$ & $\%$ & $\mathrm{n}^{0}$ & $\%$ & $\mathrm{n}^{0}$ & $\%$ & \\
\hline Gentamicina & 1238 & 96,2 & 160 & 95,2 & 268 & 97,5 & 246 & 93,2 & 303 & 95,9 & 261 & 98,9 & $0,01^{*}$ \\
\hline Amicacina & 1401 & 98,6 & 178 & 99,4 & 316 & 98,8 & 300 & 98,0 & 283 & 99,0 & 324 & 98,2 & 0,676 \\
\hline Nitrofurantoína & 1381 & 96,3 & 160 & 95,2 & 299 & 96,8 & 301 & 95,3 & 295 & 96,4 & 326 & 97,3 & 0,615 \\
\hline Ciprofloxacina & 1075 & 90,9 & 133 & 95,0 & 241 & 92,7 & 236 & 90,1 & 266 & 89,9 & 199 & 88,4 & 0,194 \\
\hline Norfloxacina & 1253 & 89,8 & 170 & 95,5 & 270 & 92,2 & 263 & 89,8 & 266 & 89,0 & 284 & 85,5 & $0,005^{*}$ \\
\hline Cefalotina** & 676 & 48,3 & 14 & 7,9 & 19 & 5,9 & 183 & 69,1 & 214 & 69,0 & 246 & 75,7 & 0,105 \\
\hline Sulfametoxazol-trimetoprima & 567 & 50,6 & 68 & 43,6 & 114 & 62,0 & 122 & 47,8 & 89 & 50,0 & 174 & 50,0 & $0,009^{*}$ \\
\hline Ampicilina $^{* *}$ & 305 & 23,4 & 1 & 0,6 & 8 & 2,8 & 62 & 24,8 & 122 & 41,4 & 112 & 38,0 & $<0,001^{*}$ \\
\hline
\end{tabular}

*p $<0,05$, **:Somente analisados os anos 2003, 2004 e 2005.

Tabela 2 - Perfil de sensibilidade (\%) de Klebsiella pneumoniae, Proteus mirabillis, Pseudomonas aeruginosa e Enterobacter aerogenes nas infecções do trato urinário de pacientes ambulatoriais do Hospital Universitário de Brasília no período de 2001 a 2005.

\begin{tabular}{|c|c|c|c|c|c|c|c|c|}
\hline \multirow[b]{2}{*}{ Antibióticos } & \multicolumn{2}{|c|}{$\begin{array}{c}\text { Klebsiella } \\
\text { pneumoniae }\end{array}$} & \multicolumn{2}{|c|}{$\begin{array}{c}\text { Proteus } \\
\text { mirabillis }\end{array}$} & \multicolumn{2}{|c|}{$\begin{array}{c}\text { Pseudomonas } \\
\text { aeruginosa }\end{array}$} & \multicolumn{2}{|c|}{$\begin{array}{c}\text { Enterobacter } \\
\text { aerogenes }\end{array}$} \\
\hline & total $^{*}$ & 2005 & total $^{*}$ & 2005 & total* & 2005 & total* & 2005 \\
\hline Ampicilina & 3,6 & 5,7 & 50,0 & 60,0 & 1,7 & 0,0 & 7,0 & 20,0 \\
\hline Amicacina & 90,9 & 97,8 & 96,4 & 96,7 & 67,6 & 75,0 & 95,0 & 94,4 \\
\hline Cefalotina & 55,9 & 42,9 & 72,5 & 76,0 & 0,0 & 0,0 & 13,6 & 17,6 \\
\hline Ciprofloxacina & 89,8 & 91,7 & 94,3 & 100,0 & 60,3 & 58,3 & 83,6 & 81,3 \\
\hline Gentamicina & 86,3 & 88,2 & 91,4 & 91,7 & 55,6 & 58,3 & 90,9 & 92,9 \\
\hline Norfloxacina & 87,3 & 84,1 & 94,3 & 100,0 & 63,6 & 61,5 & 82,8 & 75,0 \\
\hline Nitrofurantoína & 73,9 & 73,3 & 11,2 & 20,7 & 1,9 & 0,0 & 58,2 & 75,0 \\
\hline $\begin{array}{l}\text { Sulfametoxazol- } \\
\text { trimetoprima }\end{array}$ & 70,4 & 76,6 & 62,4 & 60,0 & 19,6 & 23,1 & 75,0 & 81,3 \\
\hline
\end{tabular}

*Total refere-se a todo o período estudado (2001 a 2005).

\section{DISCUSSÃo}

A infecção do trato urinário é uma das infecções mais prevalentes na clínica médica, sendo o seu tratamento, na maioria das vezes, iniciado e até completado de maneira empírica. Essa conduta é justificada pelo fato da urocultura (padrão ouro) exigir até mais de seis semanas para expedir o resultado, como no caso das micobactérias ${ }^{16}$, e por ser a ITU importante causa de morbidade ${ }^{14}$.

Diante da grande incidência de falha terapêutica e visto que o tratamento inicial é empírico, impõe-se a cada serviço o conhecimento da prevalência e frequiência dos agentes e também o perfil de sensibilidade dos microrganismos aos antimicrobianos mais utilizados na prática clínica, buscando assim a otimização do tratamento e a redução do aparecimento de novas resistências.

Em nosso estudo, a Escherichia coli foi o agente mais isolado das uroculturas, representando $62,4 \%$ do total. Na literatura, encontramos que ela é o agente causal em 70\% - 95\% das ITUs adquiridas na comunidade ${ }^{714}$, corroborando nossos achados em relação à prevalência. Analisando a resposta dos antimicrobianos à Escherichia coli, observamos boa sensibilidade aos aminoglicosídeos, nitrofurantoína e quinolonas.

Estudos mostraram a relação entre uso anterior de antibióticos e aumento da resistência da Escherichia coli à ampicilina e sulfametoxazol-trimetoprima ${ }^{13}$. Corroborando com a informação acima, a prescrição de trimetoprima seis meses antes foi 2,5 vezes mais comum no grupo que apresentou resistência à droga ${ }^{20}$. Portanto, o uso racional de antibióticos, respeitando dose e tempo de tratamento, e o conhecimento dos agentes mais freqüentes e dos respectivos perfis de sensibilidade na comunidade são mandatórios, visto a necessidade de se evitar falhas terapêuticas e seleção de microrganismos resistentes ${ }^{14}$.

Foi observado também em nosso estudo baixa sensibilidade da Escherichia coli à STX-TMP. 0 guia de terapia antimicrobiana Sanford $2005^{17}$ registra que a resistência da Escherichia coli ao STX-TMP é alta (15\%-20\%) e está correlacionada com falha no tratamento. A recomendação é que em áreas onde a resistência local é menor que 20\%, a terapêutica com STX-TMP é recomendada; porém, em áreas onde a resistência local é maior que $20 \%$, a fluoroquinolona deve ser usada ${ }^{14}$.

Nos últimos anos, a resistência à STX-TMP tem aumentado muito, enquanto a suscetibilidade às fluoroquinolonas permanece elevada ${ }^{14}$.

Depois de uma década de uso na terapia das ITUs não complicadas, as fluoroquinolonas, entre elas a ciprofloxacina, têm mantido boa atividade contra Escherichia coli e outros patógenos implicados na etiologia das ITUs, elevando as chances de sucesso no tratamento $0^{14}$.

Um grupo de estudo do Hospital Universitário Professor Edgard Santos - UFBA, observou um aumento na frequiência 
de Klebsiella pneumoniae e Proteus mirabillis resistentes à norfloxacina e ciprofloxacina, sendo as modificações mais acentuadas na resistência em relação à norfloxacina. No entanto, a Escherichia coli apresentou pequena modificação de sensibilidade aos mesmos.

Em pesquisa anterior, feita no HUB para verificação do perfil de sensibilidade dos agentes mais comuns nas ITU em regimes ambulatorial e hospitalar, mostrou já em 1996 que a associação sulfametoxazol-trimetoprima, até então o tratamento de primeira linha, apresentou $50 \%$ de resistência ${ }^{14}$.

Em conclusão, a Escherichia coli foi a bactéria mais isolada nas ITUs comunitárias, sendo seu perfil de sensibilidade muito importante na escolha do tratamento empírico. Recomenda-se o uso empírico de quinolonas (ciprofloxacina, norfloxacina) ou nitrofurantoína, por ser a administração oral a mais utilizada, visando melhor aceitabilidade e adesão ao tratamento. Nos casos mais graves que demandam administração parenteral, os aminoglicosídeos apresentam grande efetividade. Visto que a resistência à sulfametoxazol-trimetoprima se encontra acima de $20 \%$ em nosso serviço, essa não deve ser a droga de primeira escolha nas infecções urinárias comunitárias.

Trabalhos como esse devem ser realizados periodicamente, devido a possíveis mudanças no perfil de sensibilidade e nos agentes mais freqüentes das ITUs comunitárias. Assim, será possível prover uma melhor assistência à comunidade e garantia de resolução da maioria das infecções do trato urinário.

\section{REFERÊNCIAS}

1. Andriolo A. Guia de Medicina ambulatorial e hospitalar de Medicina Laboratorial. Manole, São Paulo, 2005.

2. Correa LA, Canalini AF, Matheus WE. Etiologia das infecções do trato urinário. International Brazilian Journal of Urology 29: 7-10, 2003.

3. Fihn SD. Clinical Practise: acute uncomplicated urinary tract infections in women. New England Journal of Medicine 349: 259-266, 2003.
4. Finegold SM, Martin WJ. Diagnóstico microbiológico. Editora Médica Panamericana, São Paulo, 1983.

5. Graham JC, Galloway A. The laboratory diagnosis of urinary tract infection. Journal Clinical Pathology 54: 911-909, 2001.

6. Hooton TM. Pathogenesis of urinary tract infections: an update. Journal of Antimicrobial Chemotherapy 46: 1-7, 2000.

7. Kucheria R. Urinary tract infections: new insights into a common problem. Postgraduate Medical Journal 81: 83-86, 2005.

8. Lopes AA, Salgado K, Martinelli R, Rocha $H$. Aumento da frequiência de resistência à norfloxacina e ciprofloxacina em bactérias isoladas em uroculturas. Revista da Associação Médica Brasileira 44: 196-200, 1998.

9. Lopes HV, Tavares W. Medicina baseada em evidências. Revista da Associação Médica Brasileira 51: 301-312, 2005.

10. Marangoni DV, Moreira BM. Doenças infecciosas: conduta, diagnóstico e terapêutica. Guanabara-koogan, Rio de Janeiro, 1994.

11. National Committee for Clinical Laboratory Standards. Performance standards for antimicrobial disk sesceptibility tests. Document no M2A3, Villanova, Pa, 1984.

12. Norusis MJ. SPSS for Windows: base system user's guide. SPSS - Statistical Package for the Social Sciences inc, Chicago, 1993.

13. Pedersen G, Schonheyder HC, Steffensen FH, Sorensen HT. Risk of resistance related to antibiotic use before admission in patients with community-acquired bacteraemia. Journal of Antimicrobial Chemotherapy 43: 119-126, 1999.

14. Richard D. Rational antibiotic treatment of outpatient genitourinary infections in a changing environment. The American Journal of Medicine 118: 75-135, 2005.

15. Rosa TT, Vasconcelos D, Castro LCG, Rangel EB, Neri FAAL. Perfil microbiológico e sensibilidade a antimicrobianos de uroculturas realizadas no Hospital Universitário de Brasília, 1990 a 1992. Brasília Médica 33: 14-20, 1996.

16. Rossi F, Andreazzi DB. Resistência bacteriana: interpretando o antibiograma. Editora Atheneu, São Paulo, 2005.

17. Sanford JP. The Sanford guide to antimicrobial therapy. $35^{\text {th }}$ edition. Digital electronic version 5.0, 2005.

18. Santos SRSR, Amado CAB, Assef SMC. Infecções urinárias. Arquivos de Ciências da Saúde Unipar 3: 43-50, 1999.

19. Sato AF, Svidzinski AE, Consolaro MEL, Boer CG. Nitrito urinário e infecção do trato urinário por cocos gram-positivos. Jornal Brasileiro de Patologia Médica e Laboratorial 41: 397-404, 2005.

20. Steinke DT, Seaton RA, Phillips G, MacDonald TM, Davey PG. Factors associated with trimethoprim-resistant bacteria isolated from urine samples. Journal of Antimicrobial Chemotherapy 43: 841-843, 1999.

21. Valiquette L. Urinary tract infections in women. Canadian Journal of Urology 8: 6-12, 2001. 